\title{
Política afectiva en Las sillas de Álvaro Hernández*
}

Affective Politics in Álvaro Hernández' Chairs

Política afetiva em as cadeiras por Álvaro Hernández 


\section{Lynette Hunter**}

Distinguished Professor, History of Rhetoric and Performance, University of California Davis.

Correo electrónico: Ihunter@ucdavis.edu

Revista Corpo-grafías: Estudios críticos de y desde los cuerpos / Volumen 6 - Número 6 / Enero - diciembre de 2019 / ISSN impreso 2390-0288, ISSN digital 2590-9398 / Bogotá, D.C., Colombia / 16-37

Fecha de recepción: 3 de febrero de 2018

Fecha de aceptación: 24 de agosto de 2018

Doi: https://doi.org/10.14483/25909398.14225

Cómo citar este artículo: Hunter, L. (2019, enero-diciembre). Política afectiva en Las sillas de Álvaro Hernández. Revista Corpo-grafías: Estudios críticos de y desde los cuerpos, 6(6), 16-37 / ISSN 2390-0288.

*Artículo de investigación: El ensayo recurrirá a un performance teatral con un diseño para una audiencia reducida [Sillas]: Qué no sabemos sobre una silla vacía, producida por el dramaturgo Álvaro Hernández, para integrar este breve estudio de la política afectiva con un ejemplo de la práctica teatral.

The essay draws upon a small-audience theatre performance [Chairs]: What do we not know about an empty chair, produced by dramaturge Álvaro Hernández, to embed a brief study of affective politics in an example from theatre practice.

**Faculty member in Theatre and Dance at the University of California Davis: Much of her research work since the early 1980s has been related to the rhetoric of western democratic politics and has included many textual forms, writing genres, and performance modes. One emphasis of her current research in this area is into the various ways communities deal with diversity and create processes of valuing through performance. She has become increasingly interested in techniques relating to acting and dance, and has studied early western rhetorics of gesture and speaking, as well as training in a daoist qigong movement culture that offers alternative vocabularies for somatic change. These research areas led to Disunified Aesthetics (2014) and research into training, practice, rehearsal and performance, including a forthcoming book $A$ Politics of Practice (2018), and her current exploration of performing as training in affect. See: lynettehunteronline.com -lynettehunterperformance.com.






\section{Resumen}

La Política Afectiva se ocupa de las formas en que una persona genera devenir, conocer y valorar en un contexto situado donde los materiales son fundamentalmente no-conocidos, donde la complejidad somática del sí mismo está abierta al cambio, y cómo ese cambio se presencia en grupos colaborativos sociosituados. Este breve ensayo piensa a través de la manera en que las artes performativas están fundadas en prácticas sociosituadas donde se entrena lo afectivo, y que conducen al cambio social. Los performers no solo adquieren prácticas con materiales particulares no conocidos que fomentan los procesos afectivos para un sí-mismo procesual (selving) ${ }^{1}$ a través de formas tradicionales, sino que también aprenden prácticas que conducen a una política del escuchar más bien que a una del decir. La performatividad de esta política colaborativa ofrece soporte y sostenibilidad para lo que sucede con un grupo de personas cuando se produce un cambio. El ensayo recurrirá a un performance teatral concebido para una audiencia reducida [Sillas]: Qué no sabemos sobre una silla vacía, producida por el dramaturgo Álvaro Hernández, para integrar este breve estudio de la política afectiva con un ejemplo de la práctica teatral.

\section{Palabras clave: política afectiva; performance; materialidad, sociosituación; cambio social.}




\begin{abstract}
Affective politics is concerned with the ways a person generates becoming, knowing and valuing in a situated context where materials are fundamentally not-knowable, where the somatic complexity of a self is open to change, and how that change presences in collaborative sociosituated groups. This short essay thinks through the way that the performing arts are founded on sociosituated practices that train in affect and lead to social change. Performers not only acquire practices with particular not-known materials that encourage affective processes for selving through traditional forms, but they also learn practices that lead to a politics of listening rather than telling. The performativity of this collaborative politics offers support and sustainability for what happens with a group of people when change occurs. The essay will draw upon a small-audience theatre performance [Chairs]: What do we not know about an empty chair, produced by dramaturge Álvaro Hernández, to embed this brief study of affective politics in an example from theatre practice.
\end{abstract}

\title{
Keywords: affective politics; performance; materiality; sociosituated; social change.
}

\section{Resumo}

Política Afetiva aborda as maneiras que uma pessoa gera tornando-se, conhecer e apreciar em um contexto localizado onde os materiais são, essencialmente, como que a presença não-conhecido onde a própria complexidade somática é aberto à mudança, e mudança grupos colaborativos colaborativos. Este breve ensaio pensa em como as artes performativas são baseadas em práticas socializadas onde o afetivo é treinado, e isso leva à mudança social. Os performers não apenas adquirem práticas com materiais não familiares específicos que fomentam processos afetivos para uma selvageria processual através de formas tradicionais, mas também aprendem práticas que levam a uma política de escuta, em vez de a dizer. A performatividade dessa política colaborativa oferece suporte e sustentabilidade para o que acontece com um grupo de pessoas quando ocorre uma mudança. O julgamento vai usar uma performance teatral concebida para um público pequeno [cadeiras] não sabem sobre uma cadeira vazia, produzida pelo dramaturgo Álvaro Hernández, a integrar este breve estudo da política afetivos com um exemplo da prática teatral.

Palavras-chave: política afetiva; desempenho; materialidade, sociosituação; mudança social. 
A la política afectiva le competen las vías a través de las cuales una persona genera devenir, conocer y valorar en un contexto situado. Este breve ensayo pensará en las palabras de esa frase inicial, así como en cuatro aspectos clave de la política: que los materiales en esa ecología son desconocidos o no conocidos, que la complejidad somática del sí mismo está abierta al cambio y a la diferenciación en un proceso llamado sí-mismo procesual, que el cambio percibido es presenciado a través de estrategias incorporadas, y que la colaboración o la postura retórica de la política es un modo de relacionalidad necesariamente no relacional ya que una ecología está siempre en proceso, siempre cambiando.

Una manera de pensar sobre la acción política afectiva es atendiendo a la distinción entre el discurso sociocultural y la colaboración sociosituada, lo cual es también distinguir entre dos de los modos en que vive una persona. Las estructuras discursivas de la hegemonía liberal y neoliberal requieren "individuos" quienes estén entrenados en un accionar de causa y efecto basado en cosas cognoscibles, que sean autónomos y, por ende, limitados (sujetados dado que el sujeto es subyugado), y cuya interacción retórica (en el sentido de la persuasión social de la retórica clásica) sea dada a través de paradigmas universalistas que surgen de lógicas fundamentalistas (lógicas que no pueden ser cuestionadas). En teoría crítica, el conocimiento paradigmático y la ética estática del discurso sociocultural producen individuos "esencializados".

Sin embargo, la mayoría de nosotros también reconoce partes de nuestras vidas que no se ajustan a estos paradigmas. Al mismo tiempo que vivimos como individuos, en tanto persona también llevamos nuestras vidas al lado del discurso de maneras que a menudo tienen poco interés para el poder hegemónico, a veces invisible e inaudible para éste dado que son imposibles de reconocer; a veces activamente descartadas por éste, a veces oscurecidas, extrañadas o hechas extrañas. Estas ocurrencias paralelas a nuestro sí mismo tienen una localización situada entre grupos de personas y cosas que están involucradas en procesos que generan otras maneras de vivir. De aquí en adelante, a esto me refiero como co-laboración sociosituada o colaboración.

La política del afecto trata acerca de personas construyendo formas de seguir viviendo al lado de, en contraposición a vivir en reacción al discurso, lo cual está basado en la creación de estrategias de un sí-mismo procesual. Un sí-mismo, -en tanto no es el individuo autónomo necesitado por el discurso- tiene un límite poroso en relación a los materiales en la ecología en la cual participa. Sin embargo, este no puede saber qué son esos materiales. La apertura del sí- 
mismo está basada en no saber de modo que su ecología acontezca, sea "situada" en lugar de "hecha". Al mismo tiempo, cuanto más consciente sea el sí mismo de no saber, es más probable que una ecología situada se forme. Para trabajar con materiales no conocidos, el sí mismo debe estar atento a lo que le sucede: esto es todo lo que tenemos. Debido a que el sí mismo no puede predecir o controlar lo que ocurrirá, el atender deviene una vía de abrir el cuerpo en el modo de la escucha y su complejidad somática a los materiales que lo rodean, y de perder su autonomía dentro de una ecología particular. Por ejemplo, si recordamos que la piel es nuestro segundo pulmón, podemos llegar a tomar conciencia de las maneras de hablar, de las figuras que nos animan a pensar en el movimiento continuo que se produce a través de la piel. Yo podría decir "dentro de esta y fuera de esta" pero eso implica que el movimiento atraviesa la piel en lugar de la piel siendo completamente parte del movimiento que ocurre. A través de la piel nos encontramos en una relacionalidad porosa y transformativa (morphing), de modo que constantemente estamos tocando las cosas que nos rodean en un proceso continuo de formación de ecologías particulares.

A pesar de que el sí-mismo puede abrirse a otras cosas en una ecología particular, éste no puede conocerlas. El sí-mismo ni siquiera puede conocerse a sí, pero puede encontrar formas de devenir, conocer y valorar en su obrar paralelo. Estar abierto en una ecología significa que la porosidad del sí-mismo permite o auspicia el cambio a medida que las cosas se mueven a través de su enredo somático y éste se mueve a través de ellas. Yo llamo a este movimiento de apertura y concurrencia un proceso de formación transformativa. Si podemos pensar en la apertura a través de la figura de la piel porosa que nos conecta con nuestro ambiente, tal vez podamos pensar en el concurrir como una especie de re-membramiento, trayendo a los miembros del sí-mismo juntos de nuevo, recordando la membrana de nuestra piel $^{2}$ a través de repeticiones que no son replicaciones sino una relacionalidad constantemente variable. Así como nuestras memorias son continuamente curvadas por el presente momentáneo en el cual emergen, así, la concurrencia es continuamente afectada y llevada por la localización situada en la cual ocurre.

A diferencia del individuo que "sabe que las cosas son diferentes" a esto, el sí-mismo de una persona solo sabe que a través de la transformación, el cambio le ha ocurrido a su sí-mismo y ha llegado a ser diferenciado en cuanto tal, es decir, es ya diferente del sí mismo que comenzó atendiendo a la ecología inicial. Un individuo es un objeto y por tanto es subyugado al pertenecer a la formación de sujeto. Un sí-mismo es una cosa con una materialidad. Un sí-mismo reconoce la diferencia no en los objetos a su alrededor, sino en cómo ha devenido a través de las transformaciones que han acontecido con las materialidades en un momento particular. La diferencia no preexiste, por el contrario, es un cambio que ocurre a través de las ecologías inmersas en el acontecer.

2 Técnicamente, re-membrar puede ser 'hacer carne de nuevo' 
El afecto puede ser pensado como el sentir sentido (felt sense) de ese cambio, activando la conciencia de que algo es diferente en el sí-mismo. El sí-mismo procesual presencia el puro sentir del cambio al presenciar la diferenciación que ha sucedido en el sí-mismo. Este presenciar del afecto es un gesto hacia lo que no se conoce, a los materiales en la ecología que permiten que alguna cosa suceda y que, por tanto, el sí-mismo cambie. Todos participamos en este proceso del sí-mismo y del presenciar en nuestra vida cotidiana, aunque con relativa poca frecuencia debido a la dificultad del sí mismo de operar en un campo discursivo hecho para individuos. No somos motivados por el poder hegemónico para trabajar al lado del discurso. A menos que hayan localizaciones sociosituadas (por ejemplo, amistad) o estrategias (por ejemplo, el cuidado), generalmente trabajamos al lado de solamente en momentos extremos cuando el discurso ha sido agotado - en amor, muerte, guerra, enfermedad, nacimiento, etc. Sin embargo, los performers aprenden prácticas que incentivan procesos afectivos para el sí-mismo procesual. La tarea del performer es cambiar frente a otras personas, para mediar o hacer inmediato el afecto de materiales no conocidos. Atender a, estudiar y analizar esta mediación y sus formas por el puro sentir es lo que llamo 'estética'. El cambio es lo que la audiencia, el espectador, el observador, oyente, jugador, desea atestiguar-siendo una forma en la que el sí-mismo se abre a una ecología mediada, es un modo de prestar atención- en mayor o menor medida.

Los performers se entrenan en ciertas tradiciones. Siendo estas sostenibles solamente a través de la habilidad de sus prácticas para reformarse continuamente en localizaciones situadas. Por lo tanto, estas prácticas incentivan el obrar al lado de y el entrenamiento del afecto. Las prácticas tienen estrategias materiales específicas para abrir, concurrir, transformar, diferenciar y activar el cambio en el sí-mismo, las cuales constituyen la base del entrenamiento de una persona con sus materiales particulares. Ellas también tienen estrategias para el presenciar propio del sentir sentido del sí-mismo procesual con el objeto de conducir el afecto de cambio dentro y a través de un sí-mismo diferenciado en el trabajo improvisacional de cada performer. El trabajo es improvisacional en el sentido en que el cuerpo del performer está entrenado en prácticas tradicionales, y aprende a habitarlas jugando con estas, de manera tal que estas prácticas ponen atención a la ecología particular en la cual ocurren generando incorporaciones apropiadas para el trabajo colaborativo requerido por el performance. Contrariamente a la comprensión habitual de "tradición" como "convención estática", y más cerca al sentido indígena de "conocimiento tradicional", estas prácticas tradicionales permanecen siendo fuertes a través de los años por medio de la improvisación continua y comprometida con cada localización situada. De manera más distintiva, las prácticas tradicionales tienen estrategias para obrar en conjunto con otras y encontrar formas que puedan repetir el sentir sentido y permitirnos incorporar el afecto, aquel gesto dirigido a las cosas materiales, en el medio ambiente de un performance. Este trabajo conjunto es lo que integra los performers en un entorno político continuo de activismo relacional ${ }^{3}$. Llamamos performatividad al momento en que el performance logra transportar la energía de cambio dirigiéndose a un medio particular.

3 Brian Massumi habla de activismo relacional de modos sugestivos (2011, 1-28). 
Podemos aprender mucho sobre la más amplia política del afecto considerando cómo el performance y la performatividad toman lugar cuando los performers colaboran haciendo el trabajo al lado de.

Los performers están involucrados en estrategias de atención, de escuchar en lugar de decir, que conducen no solo al proceso del sí-mismo procesual de involucrarse con los materiales, sino también a la incorporación que ocurre a través de la habilidad de la improvisación, y a través de la postura retórica o relacionalidad basada en la co-laboración en el taller, estudio, ensayo y laboratorio ${ }^{4}$. Cuando el sí-mismo procesual se mueve hacia el presenciar y la co-laboración, formas de devenir y conocer se desplazan hacia el valorar.. El afecto marca todos los tres procesos. La colaboración es una política que genera posibilidades de cambio en una localización situada, más o menos probable de permitir otros procesos del sí-mismo hacia ese cambio. Es una relacionalidad política que ofrece el soporte de otras personas en el caso de que ocurra cualquier vulnerabilidad en ese cambio. $Y$ en las tradiciones del performance de persona a persona, ofrece apoyo para aquello que sucede en el trabajo de grupo y cómo es incorporado - su posicionalidad.

Estas tradiciones no solo apoyan la incorporación de un proceso del sí-mismo procesual hacia una posicionalidad, sino que también entrenan al performer en evaluar si vale la pena el costo de la reducción, el cual siempre es alto cuando se establece la posicionalidad de un grupo en una forma discursiva emergente. El performer podría optar por la forma que es "más probable" para incorporar una posicionalidad, o por una en la que sea "más probable" reducirla a un direccionamiento hacia el discurso. Al hacer lo uno u lo otro, los performers permiten que una audiencia participe de la ecología de la performatividad de distintas maneras. Una posicionalidad colaborativa alienta a la audiencia atestiguante a involucrarse en lo sociosituado con el material no conocido, mientras que un direccionamiento hacia el discurso les ayuda a articular ese material hacia lo sociocultural. En el último caso con sus estrategias asociadas en colectividad, consenso, lo corporativo, y más - todo opera como si la diferencia ya estuviera allí. La colaboración funciona con la sensación de que la diferencia sucede y que los co-laboradores trabajen enfocados en cómo presenciar lo que sucede. Si el sí-mismo procesual es la diferenciación que le acontece a una persona cuando pierde su autonomía y se transforma con la ecología del momento, y si el afecto es el sentir sentido de ese sí-mismo, entonces sus políticas están enraizadas en las estrategias improvisacionales para presenciar ese cambio a través de la colaboración.

Para volver al principio: este breve ensayo intenta reflexionar sobre la manera acerca de cómo performers, a través de la colaboración, posibilitan una política afectiva en la cual otros cambian, y al hacer eso, toman conciencia de

\footnotetext{
4 Yo uso la palabra 'engaging' (aquí involucrarse) aquí y a lo largo de este ensayo, para significar la vía en que el proceso de transformación atrae al sí-mismo hacia los materiales a su alrededor. Involucrarse es transformarse con las otras cosas en la localización. En estudios literarios esto ha sido llamado tradicionalmente 'alegoría' (Hunter, 2010), y siempre se ha enfocado en la participación del lector en la textualidad, así que deviene una textualidad situada. En los estudios del performance, esta es central para el obrar de la performatividad. Karen Barad también usa 'engaging' de maneras similares (2001, 75-109)
} 
formas alternativas de devenir, conocer y valorar. El núcleo de esta política radica en la forma en que las prácticas del performer se involucran con cosas en tanto no conocidas y abiertas al sentir sentido del sí- mismo diferenciado que en consecuencia se produce. En el ensayo, el performer intenta presenciar el afecto de ese sentir sentido a través de estrategias incorporadas tales como la improvisación. El grupo a su alrededor co-labora en estas repeticiones a lo largo del proceso de ensayo de modo que la audiencia pueda comprometerse con la materialidad del medio que han creado. Todas estas prácticas están en una continua relacionalidad no relacional, sin embargo, la repetición del afecto a través de las formas y dentro de un medio estabiliza la colaboración más que el presenciar, y el presenciar más que el sí-mismo procesual. Esta es la forma en que funciona el performance -transportando el afecto a través de las formas que emergen.

Me gustaría ahora referirme a un performance que he atestiguado, Sillas, del dramaturgo y director colombiano Álvaro Hernández, que enfatiza en las relacionalidades del presenciar y la co-laboración. La pieza extiende las prácticas de ensayo dentro del performance, atrayendo al público directamente hacia su ecología. Las sillas, se llevó a cabo inicialmente en una jornada de 6 performances en septiembre de 2017. En el espacio de juego escénico había 5 actores, un camarógrafo, 2 técnicos y 14/15 miembros de la audiencia para cada performance. Yo asistí a estas presentaciones y a dos ensayos. Hernández trabaja con materiales que, para él, y uno siente que también para el elenco, transportan el afecto de muerte: un puro sentir de la ausencia / presencia de la muerte, particularmente de muertes inciertas, de aquellos que han desaparecido. El título completo de la pieza es: “¿En lo que es desaparecer? Lo que no sabemos acerca de una silla vacía ", y la tensión en el drama surge de las cualidades no conocidas de la muerte, de aquellos que han muerto o podrían haber muerto, y de los objetos que nos los recuerdan incluso sin conocerlos.

El área de juego en un antiguo sótano reutilizado se encontraba al principio vacía. Los miembros de la audiencia fueron conducidos escaleras abajo y parados a lo largo de dos de las paredes mientras un extenso rollo de cuerda gruesa era jalado ocupando el espacio. Rápidamente el área se llenó con objetos domésticos como cuchillos y tenedores, platos, fotografías, juguetes de peluche, y con sillas y taburetes, en algunos de los cuales se invitó a los miembros del público a sentarse.

Durante algo menos de una hora, las partituras de la pieza se desplegaron a su alrededor mientras los intérpretes jugaban con los objetos, improvisando sobre las prácticas en las que están entrenados: movimiento, canto, video, voz, palabra hablada y acción corporal, entre otros. 


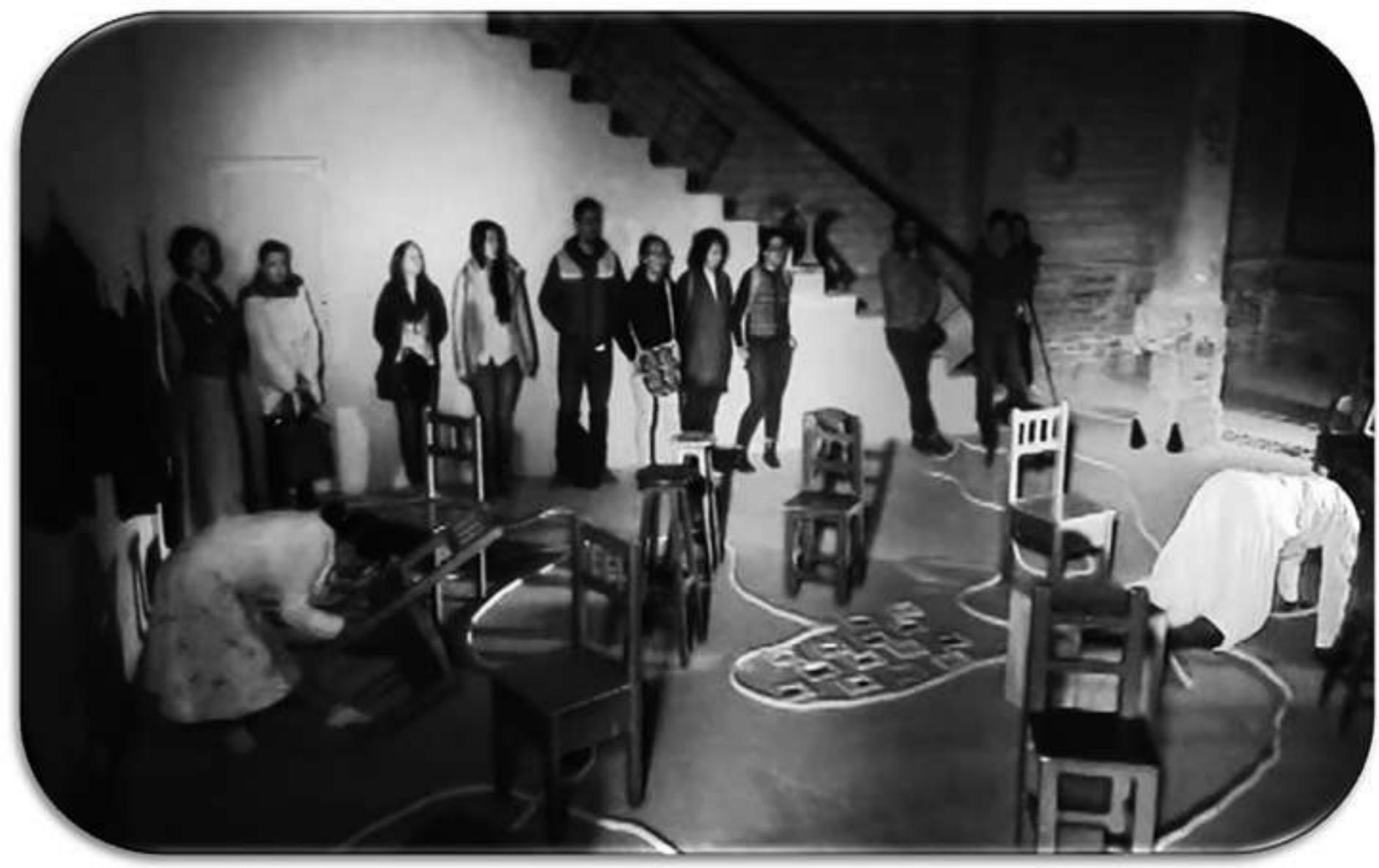

Figure 1. Chairs and Objects: from Alvaro Hernández' Chairs... Photo: Alvaro Hernández, 2016.

Los ensayos incitaron a los performers a trabajar una y otra vez con los objetos a su alrededor y a desarrollar prácticas que podrían mantener el sentir de estos objetos como no-conocidos. Por ejemplo, un performer creó una partitura alrededor del sentarse en una silla en docenas de maneras distintas, y así, de repente, estaba tan solo sentándose / sentado en la silla, como si fuera molecularmente uno con ella, atrayendo una intensa conciencia de que un cambio en el espacio había sucedido. A veces, esa intensidad era sensación /sentida surgida de un movimiento poderoso y veloz. Un intérprete levantó una silla en lo alto muy por encima de su cabeza y, casi corriendo, la sacudió con fuerza hacia abajo, -algo potencialmente peligroso en el espacio lleno con 14 participantes de la audiencia y 5 actores presentes, - más en esencia se trataba de un momento de quietud guiado formalmente, una forma ensayada que transportaba un afecto de ira y / o frustración. Cada intérprete se involucró con la forma, la resiliencia, el peso de las sillas. Como miembro de la audiencia, uno podría sentir que también cada uno asistía a la forma en que una silla o taburete particular liberaba un impulso en su complejidad somática, desde "cómo no" dejar caer 'la silla' hasta la 
casi excitación erótica en su textura. Sus movimientos-con-la silla presenciaban ese impulso, no como una especie de réplica, sino como una improvisación construida sobre una serie de rememoraciones sucediendo en y alrededor del cuerpo del performer en el momento inmediato del performance. Este afecto incorporado nos condujo hacia nuestro propio compromiso con la materialidad que nos rodeaba.

Estos momentos le sucedían a cada performer en la ecología más amplia de un grupo de personas co-laborando y apoyándose mutuamente. Si estos ejemplos previos son de performers solistas moviéndose con objetos, también se dieron otros momentos en que los performers trabajaron juntos directamente. En un momento dado, dos intérpretes "casi" se sientan en la misma silla, como si jugaran con una u otra posibilidad; pero performance tras performance, aunque yo seguía sintiendo que podría ser uno u otro, siempre fue el mismo intérprete el que se "sentaba :

En otro instante, un performer, quien tuvo que faltar a varios de los ensayos finales, llegó momentos antes de que se abriera el espectáculo y éste fue guiado en ensayo a través de movimientos particulares formados que tenían tiempos precisos. Apenas pude adivinar que la mayoría de las partituras de movimiento tenían esta estructura formal sin importar cuán inmediatas se sintieran. Pero entonces, la inmediatez es un afecto de la improvisación.

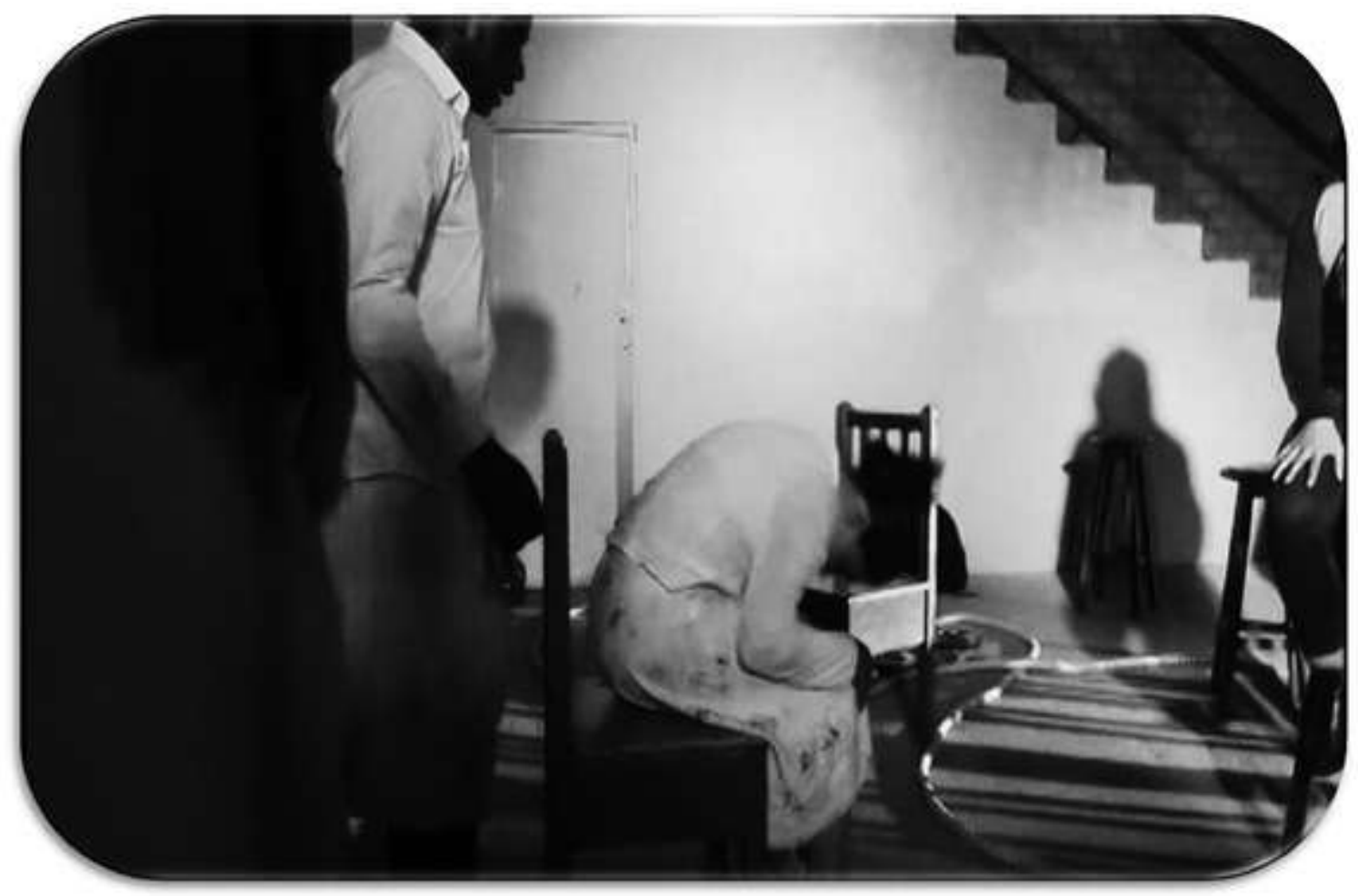

Figure 2. Sitting: from Alvaro Hernández' Chairs... Photo: Alvaro Hernández, 2016. 
La partitura visual de la proyección de video era aparentemente más formada / diseñada a través de su mediación por software, más sin embargo también fue claramente improvisada. Había una retroproyección en una pantalla a lo largo de un lado del área de actuación la cual cuando se alimentaba desde una cámara de video colocada a la derecha del área, mostraba la imagen especular de lo que estaba sucediendo. Simultáneamente se generaba una proyección granulada de cualquier imagen que se estuviera reproduciendo a través de la pantalla y sobre la pared opuesta.

Al mismo tiempo, detrás de la pantalla y en la pared posterior del área elevada, se daban extraños efectos de luz tenue, como imágenes estriadas. Parte del video presentaba las acciones ocurridas en el espacio de actuación principal a través de un programa que disolvía las imágenes de los cuerpos de los actores cada vez que se movían. La partitura del video continuaba improvisando en torno a la idea de la realidad visual, utilizando el entorno digital para superponer capas y dispersar los bordes de los jugadores frente a nosotros, generando la sensación de que nunca se



Figure 3. Projections: from Alvaro Hernández' Chairs... Photo: Alvaro Hernández, 2016. 
podía ver / escuchar / sentir lo que realmente estaba sucediendo, que lo inmediato tenía una calidad déja vue que se deslizaba entre los dedos.

La partitura de sonido ofrecía otras cualidades improvisadas a las capas formales de la pieza. Cuando los performes comenzaron a colocar sillas y taburetes en el espacio de la acción, al principio, cantaban 'mañanita', una canción al despertar del día, Ilena de una energía que generaba olas de expectación a la audiencia. La canción se tornó hacia un tono cantado, y varios ruidos, y luego su intensidad cambió hacia un sonido cercano a los gritos. En una parte, gran porción de la cual es un regalo de la posibilidad de re-membrar, los sonidos se trasladaron de lo reconocible a lo ambiguo, al escuchar un tenue llamado de duelo. La partitura sonora transitó a través de cantos, murmuraciones, susurros, presentaciones, habla - una susurración de ruido en parte presente, parte presenciando, parte eludiendo, y finalizando con la tonada para niños de un "chigualo", una canción de una tradición afrocolombiana que se canta cuando un niño muere. La forma de la canción es la de una 'ronda', cuyas distintas voces superpuestas creaban un tejido de disonancia afectiva ${ }^{5}$, que nos juntaba a todos allí en ese lugar.

La partitura de sonido estaba estrechamente entrelazada con la partitura hablada. Hernández es escritor, poeta y dramaturgo, así que las palabras en español que yo no hablo o que apenas entiendo parcialmente, habían sido, sin embargo, elaboradas para trabajar con lo no dicho, de modo que la materialidad verbal se plegó en sonido y resonancia. Por ejemplo, cerca del final de la pieza, la historia de un padre desaparecido fue contada por una performer quien primero se movía de una silla a otra mientras hablaba. Al mismo tiempo, todos los demás performers reposicionaban a los miembros de la audiencia en un extremo del espacio de interpretación y luego corriendo a través del área movían todas las sillas hacia el extremo opuesto formando una gran pila, excepto por cuatro en las cuales ellos y quien hablaba venían a sentarse. Una vez completado, el quinto performer iniciaba una lucha con una cuerda que yacía expuesta y rodeando a todos los objetos, de vuelta hacia el espacio desde el cual se había desenrollado.

Esta acción halaba todas las cosas que se hallaban en el piso aplastando y chocando unas con otras, arrastrándose a lo largo del suelo, cayendo sobre las sillas vacías, de modo que las palabras que estaban siendo dichas no podían ser claramente oídas. La forma de la historia era improvisada sobre el torrente de actividad y el sonido generado por los colaboradores de quien hablaba, cosa que ocurría solo en partes y pedazos.

Nosotros los miembros de la audiencia fuimos sorprendidos por palabras que podían oírse, tonos que eran casi familiares, más sin poder saber cuál era la historia -del mismo modo que el narrador no podía conocer al desaparecido.

5 Ver la relacionada 'disonancia simpatética' (Bolles and Hunter, 2012, 153-68). 


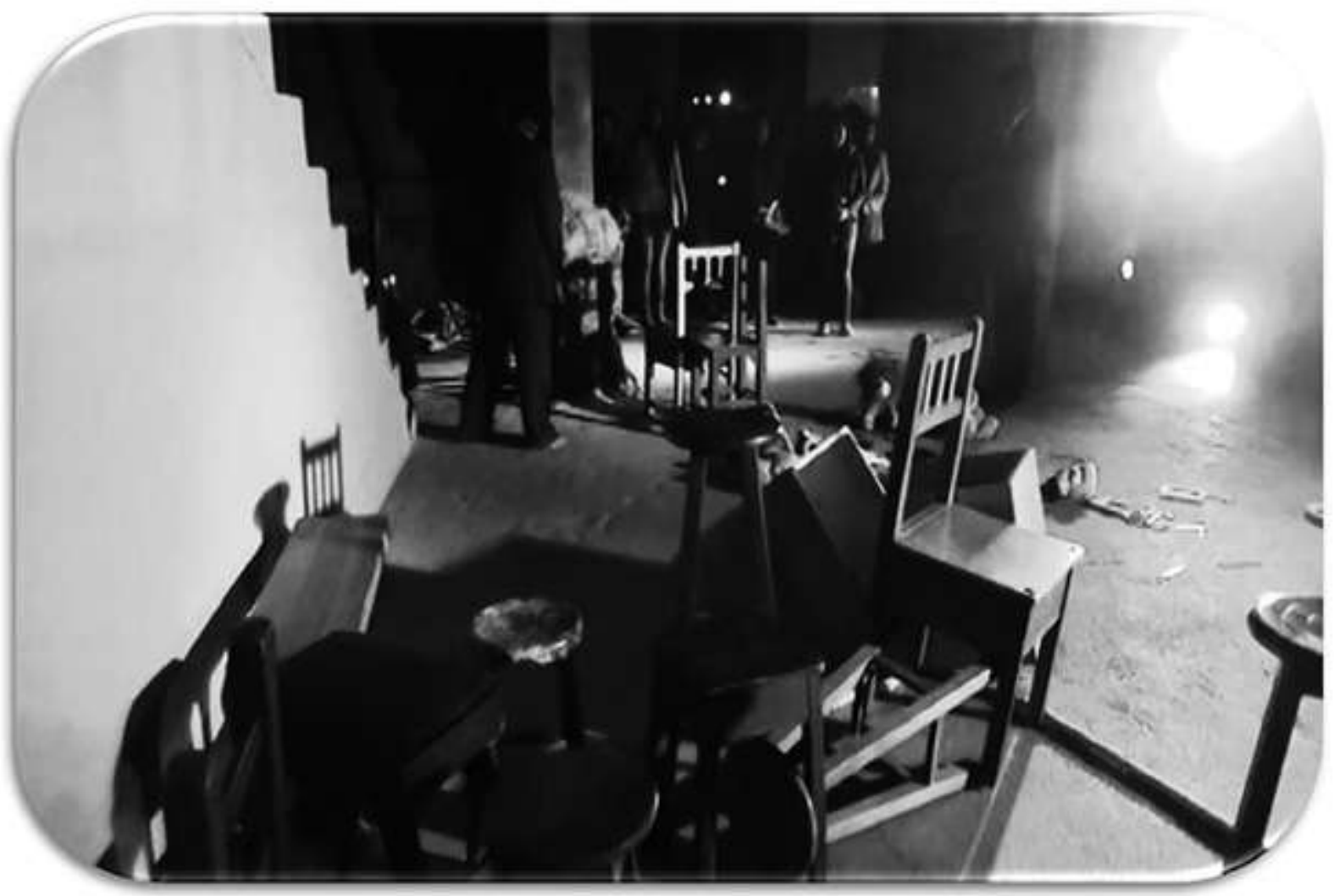

Figure 4. Crushed Objects: from Alvaro Hernández' Chairs... Photo: Alvaro Hernández, 2016.

No se puede garantizar si y como un miembro de la audiencia se llega a involucrar con este material incorporado e incognoscible. Sin embargo, en esta pieza el continuo presenciar a través de la improvisación sobre formas reconocidas aunque inesperadas, como objetos domésticos y cosas cotidianas, las colaboraciones constantes entre los performers, la conexión entre ellos y con todos los materiales en el espacio, y la habilidad para interpretar estos dentro de las partituras que transmitían el afecto, hacían más probable que nos diéramos a co-laborar con el proceso. El uso de estrategias de ambigüedad, elusividad, deslizamiento, disonancia, con practicantes altamente calificados, hacía también mucho más probable el construir una localización sociosituada que generaría una posicionalidad con la que el público también colaboraría. Pero también sería muy probable el reducir esto a un significado sociocultural.

Colaborar en ese proceso de 'no-saber' es lo que anteriormente llamé generar una posicionalidad. Es difícil hablar de compromisos de la audiencia que no sean los míos propios, pero Hernández le pidió a la audiencia que se tomara el tiempo, al salir del edificio, para escribir en un cuaderno acerca de sus sensaciones. Algunos encontraron frustrante 
la pieza y no sabían qué hacer con ella, ni cómo articular su "significado". Otros la encontraron profundamente afectiva - los cambió. La obra los condujo hacia su entramado, les pidió dejar de lado su individualidad, y les ofreció materiales para su propio olvido y recuerdo, su propio sí-mismo procesual que reconoce los muertos en el cambio que acontece mientras co-laboran como miembros de la audiencia con el performance. Al hacer esto, se unieron a la posicionalidad de la política afectiva. El Performance que trabaja para sustentar el afecto de una materialidad con la cual el público puede co-laborar está incorporando una política afectiva fundamental para la performatividad. También es fundamental para la colaboración sociosituada que genere las posicionalidades necesarias para soportar y sostener el cambio social a largo plazo - a través del camino la diferencia le sucede al sí-mismo y el sí-mismo comienza a sentir otras maneras de devenir, conocer y valorar.

Versión en inglés

Affective politics is concerned with the ways a person generates becoming, knowing and valuing in a situated context. This short essay will think about the words in that opening sentence, as well as four key aspects of the politics: that the materials in that ecology are unknowable or not-known, that the somatic complexity of the self is open to change and to differentiation in a process called selving, that the felt change is presenced through embodied strategies, and that collaboration or the rhetorical stance of the politics is a mode of relationality necessarily non-relational because an ecology is always in process, always changing.

One way to think about affective political action is to attend to the distinction between sociocultural discourse and sociosituated collaboration, which is also to distinguish between two of the ways that a person lives. The discursive structures of both liberal and neoliberal hegemony require "individuals" who are trained in cause-and-effect action based on knowable things, who are autonomous and hence bounded (and bound, as the subject is subjected), and whose rhetorical (in the sense of the social persuasion of classical rhetoric) interaction is through universalist paradigms arising from fundamentalist assumptive logics (logics that cannot be questioned). In critical theory, the 
paradigmatic knowledge and static ethics of sociocultural discourse result in "essentialized" individuals. Yet most of us also acknowledge parts of our lives that do not fit these paradigms. At the same time as we live as individuals, as a person we also carry out our lives alongside discourse in ways often of little interest to hegemonic power, sometimes unseen and unheard by it because they are impossible to recognize, sometimes actively discarded by it, sometimes obscured, sometimes estranged or "made strange." These alongside occurrences of our "self" have a location situated among groups of people and things that are involved in processes that generate other ways of living. This I refer to as sociosituated co-laboring or collaboration.

If the politics of affect is about people building ways of going on living alongside rather than in reaction to discourse, it is based on a process of selving. A self, because it is not the autonomous individual needed by discourse, has a porous boundary with the materials in the ecology in which it participates, yet it cannot know what those materials are. A self's openness is based on not-knowing so that its ecology happens, is "situated" rather than is "made." At the same time the more a self is aware of not-knowing, the more likely a situated ecology will form. To work with not-known materials, the self has to be attentive to what happens to its-self: this is all we have. Because the self cannot predict or control what will occur, attending becomes a way of opening the sounded body and its somatic complexity to its surrounding materials, and of loosing its autonomy into a particular ecology. For example, if we remind ourselves that the skin is our second lung, we can become aware of ways of speaking, of figures that encourage us to think about the continual movement going on through the skin. I could say "into it and out of it," but that implies that the movement crosses the skin rather than the skin being wholly part of the movement that happens. Through the skin we are in a porous, morphing relationality so that we are constantly touching things around us in an on-going process of forming particular ecologies.

Even as the self can open to other things in a particular ecology, it cannot know them. The self cannot even know itself, but it can find ways of becoming, knowing and valuing in its work in the alongside. Being open in an ecology means that the porousness of self enables or hosts change as things move through its somatic tangle and it moves through them. I call this movement of opening and gathering a morphing process. If we can think of opening through the figure of the porous skin that connects us to our environment, perhaps we can think of gathering as a kind of re-membering, bringing the members of the self back together, remembering the membrane of our skin, ${ }^{6}$ through repetitions that are not replications but a constantly varying relationality. Just as our memories are continually inflected by the momentary present into which they emerge, so gathering is continually inflected and charged by the situated location in which it occurs. 
Unlike the individual which "knows that things are different" to it, a person's self knows only that through morphing, change has happened to their self and it has become differentiated, different to the self that began attending to the initial ecology. An individual is an object, and hence bound to the subject. A self is a thing with a materiality. A self recognizes difference not in the objects around it but in how it has itself become different through morphing with the materialities in the particular moment. Difference does not pre-exist, it is a change that occurs through ecologies of happening.

Affect can be thought of as the felt sense of that change, an awareness that something is different in the self than before. Selving presences that felt sense of change by presencing the differentiation that has happened in the self. This presencing of affect is a gesture toward what is not-known, to the materials in the ecology that enabled some thing to happen, and the self to change. We all engage in this selving and presencing process in our everyday lives, although relatively infrequently because of the self's difficulty of operating in a discursive field made for individuals. We are not encouraged by hegemonic power to work alongside discourse. Unless there are sociosituated locations (eg friendship) or strategies (eg caring), we usually work in the alongside only at extreme moments when discourse has been exhausted - in love, death, war, illness, birth etc. However, performers learn practices that encourage affective processes for selving. The performer's task is to change in front of other people, to mediate or make immediate the affect of not-known materials - attending to, studying and analyzing this mediation and its forms for felt sense is called "aesthetics." Change is what the audience, spectator, viewer, listener, gamer, wants to witness - witnessing, being a way that the self opens to a mediated ecology, is a mode of attending - to a greater or lesser extent.

Performers train in traditions, a tradition only being sustainable through the ability of its practices continually to reform in situated locations, hence these practices encourage alongside work and training in affect. The practices have material-particular ${ }^{7}$ strategies for opening, gathering, morphing, differentiating, and selving, which make up the groundwork of a person's training with their particular materials. They also have strategies for presencing the felt sense of selving, for carrying the affect of change into and through a differentiated self in the highly skilled improvisational work of each performer. The work is improvisational in the sense that the performer's body is trained in traditional practices, and learns to inhabit them by playing with the practices so they attend to the particular ecology in which they occur and generate embodiments appropriate to the collaborative work needed for performance. Counter to the usual understanding of "tradition" as "static convention," and closer to the indigenous sense of "traditional knowledge," these traditional practices remain strong over the years through continual and engaged improvisation with each situated location. Most distinctively, traditional practices have strategies for working together with others 
to find forms that can repeat the felt sense and enable us to embody the affect, that gesture to material things, into a medium of performance. This working together is what embeds performers in an ongoing political environment of relational activism. ${ }^{8}$ When the performance carries the energy of change into a medium we call it performativity.

We can learn a lot about the wider politics of affect by considering how performance and performativity play out when performers collaborate in making work.

Performers are engaged in strategies of attending, of listening rather than telling, that lead not only to the selving process of engaging with materials, but also to the embodying that occurs through the skill of improvisation, and through the rhetorical stance or relationality that is based in co-laboring in workshop, studio, rehearsal, and laboratory. ${ }^{9}$ When selving moves into presencing and co-laboring, ways of becoming and knowing move also into valuing. Affect marks all three processes. Collaboration is a politics that generates possibilities for change in a situated location, more or less likely to enable other selving processes toward that change. It is a political relationality that offers other people support for any vulnerability in that change, and in the performance traditions of person-withperson activity it offers support for what happens in the group work and how it is embodied - its positionality.

These traditions not only support the embodiment of a selving process into positionality, but also train the performer in evaluating whether the cost of reduction, which is always high when setting the positionality of a group into an emergent discursive form, is worth it. The performer could go for the form that is "more likely" to embody a positionality, or one that is "more likely" to reduce it to a set toward discourse. In doing one or the other they enable an audience to attend to the ecology of the performativity in distinct ways. A collaborative positionality encourages the witnessing audience to engage in the sociosituated with not-known material, while a set toward discourse helps them to articulate that material into the sociocultural. The latter - with its associated strategies in collectivity, consensus, the corporate, and more - all operate as if difference is already there. Collaboration works with a sense that difference happens. And that the co-laborers work on how to presence what happens. If selving is the differentiation that happens to a person when they lose their autonomy and morph with the ecology of the moment, and if affect is the felt sense of that selving, then its politics are rooted in the improvisational strategies for presencing that change through collaboration.

\footnotetext{
8 Brian Massumi speaks of relational activism in suggestive ways (2011, 1-28).
}

9 I use the word 'engaging' here, and throughout, to signify the way that the morphing process draws the self into the materials around it. To engage is to morph with the other things in the location. In literary studies this has traditionally been called 'allegory' (Hunter, 2010), and has always focused on the reader's participation in textuality, so that it becomes a situated textuality. In performance studies, it is central to the work of performativity. Karen Barad also used 'engaging' in similar ways (2001, 75-109). 
To return to the beginning: this short essay is thinking about how performers, through collaboration, enable an affective politics in which others change and in doing so become aware of alterior ways of becoming, knowing, and valuing. The core of this politics lies in the way the performer's practices engage with things as not-known and open to the felt sense of the differentiated self that then comes about. In rehearsal, the performer attempts to presence the affect of that felt sense through embodied strategies such as improvisation. The group around them co-labours on these repetitions throughout the rehearsing process so that an audience may engage with the materiality of the medium they create. All of these practices are in a continual non-relational relationality, yet the repetition of affect through forms and into a medium stabilizes collaboration more than presencing, and presencing more than selving. This is the way performance works - carrying affect through the forms that emerge.

I would like now to turn to one performance I have witnessed, Chairs by Colombian playwright and director Álvaro Hernández, which dwells on the relationalities of presencing and co-laboring. The piece sustains the rehearsal practices into the performance, drawing the audience directly into its ecology. Chairs took place initially over six performances in September 2017. In the playing space there were 5 actors, one videographer, 2 technicians, and 14/15 audience members for each performance. I attended these performances and two rehearsals. Hernández works with materials that for him, and one senses, for the cast, carry the affect of the dead - a felt sense of the absence/presence of death, particularly of uncertain deaths, of those who have disappeared. The full title of the piece is: "On What Is It to Disappear? What We Not Know about an Empty Chair," and the tension in the dramaturgy arises from the not-known qualities of death, of those who have or might have died, and of objects that remind us of them even while we do not know them.

The playing area in an old re-purposed basement was at first empty. The audience members were led down the stairs, and stood along two of the walls as a large coil of thick rope was pulled into the space. But the area was quickly filled with domestic objects such as knives and forks, plates, photographs, soft toys, and with chairs and stools, on some of which the audience members are invited to sit.

For the next near-hour the scores of the piece unfurl around them as the performers play with the objects, improvising on the practices in which they are trained - movement, song, video, voice, spoken word, and bodily action, among others.

The rehearsals encouraged the performers to work again and again with the objects around them and develop practices that could sustain the sense of these objects as not-known. For example, one performer created a score of sitting in a chair in dozens of distinct ways, and then, suddenly, they were just sitting/seated into the chair, as if molecularly at one with it, drawing an intense awareness that a change in the space had happened. At times that 
intensity was felt/sensed in swift powerful movement. One performer lifted a chair high above their head and, almost running, swung it firmly down - potentially dangerous in the crowded space with 14 audience participants and 5 actors present - yet at its core it was a still moment of a formal pathway, a rehearsed form that carried an affect of anger and/or frustration. Each performer engaged with the shape, resilience, weight of the chairs - $\underline{\text { if as }}$ an audience member you attended, you could sense each one also attending, to the way a particular chair or stool released an impulse in their somatic complexity - from "how not to drop" the chair to an almost erotic arousal in its texture. Their movement-with-the-chair presenced that impulse, not as a kind of replication but as a improvisation built upon a series of re-memoryings happening in and around the performer's body in the immediate moment of performance. This embodied affect drew us into our own engagement with the materiality around us.

These moments were happening to each performer in the larger ecology of a co-laboring group of people who were each supporting each other. If these previous examples are of solo performers moving with objects, the performers also worked directly together. At one point, two performers "almost" sit on the same chair, as if playing with the possibility of either/or - but performance after performance, even though I kept feeling it could be either, it was always the same one performer who "sat."

In another instance, one performer, who had had to miss several of the final rehearsals, arrived just before the show opened and was rehearsed through particular formed movements that had precise timings. I could only guess that most of the shapes of the movement score had this formal structure no matter how immediate they felt. But then, immediacy is one affect of improvisation.

The visual score of the video projection was apparently more formed/shaped through its software mediation, yet it too was clearly improvising. There was a back-projection onto a screen along one side of the playing area which, when it fed from a camcorder positioned to the right of the area, showed the mirror image of what was happening. But there was also a grainy projection of whatever image it was playing, through the screen and onto the opposite wall.

At the same time, behind the screen and on the back wall of the raised area, were odd wispy lighting effects, like striated images. Some of the video was presenting the actions in the main acting space through a program that dissolved the images of the actors' bodies whenever they moved. The video score kept improvising around the idea of visual reality, using its digital medium to layer onto and disperse the edges of the players in front of us, generating a sense that you could never see/hear/feel what was actually happening, that the immediate had a déja vue quality slipping through your fingers. 
The sound score offered other improvised qualities to the formal layers of the piece. As the performers began to position chairs and stools in the playing space at the beginning, they were singing "mañanita" - a song to the morning, full of energy that sent waves of expectation out into the audience. The song moved into a sung tone, and various noises, and then intense song sounds almost like screams. In a piece, much of which is a gifting of the possibility of re-membering, the sounds moved from the recognizable into the ambiguous, half-heard callings-out of mourning. The sound score travelled on through chant, murmuring, whispering, addressing, speaking - a sussuration of noise part-present, part presencing, part eluding. And ended with the children's song of a "chigualo," a song from an Afro-Colombian tradition that is sung when a child dies. The form of the song is a "round," whose distinct overlapping voices created a fabric of affective dissonance, ${ }^{10}$ pulling together all of us there in that place.

The sound score was closely interwoven with the word-score. Hernández is a writer, a poet, and dramaturge, so the words in the Spanish that I don't speak or even partially understand, had nevertheless been crafted to work with the not-said so the verbal materiality folded into sound and resonance. For example, near the end of the piece, the story of a disappeared father was told by one performer who was at first moving from chair to chair as they spoke. At the same time all the other performers were re-positioning the audience members at one end of the playing space, and then running through the area moving all the chairs to the opposite end and into a huge pile, except for four on which they and the speaker came to sit. Once completed, the fifth performer began to wrestle a rope that lay exposed and encircling all the objects, back into the space from which it had been uncoiled. This action pulled all the things on the floor crushing and crashing together, scraping along the ground, toppling over the empty chairs, so that the words being spoken could not clearly be heard. The form of the story was improvised upon by the torrent of activity and sound generated by the speaker's collaborators, happening only in pieces and parts.

We the audience members were surprised by words that could be heard, tones that were almost familiar, yet could not know what the story was - just as the teller could not know the disappeared. How an audience member engages with this not-known embodied material is never guaranteed. Yet in this piece the continual presencing through improvisation on recognized if unexpected forms like domestic objects and day-to-day things, the constant collaborations among the performers, their awareness of each other and all the materials in the space, and skill in playing these into scores that carried the affect, was more likely than not to pull us into co-laboring with the process. Using strategies of ambiguity, elusiveness, slippage, dissonance, with highly skilled practitioners, was not only more likely than not to build a sociosituated location that would generate a positionality with which the audience would also collaborate, but also more likely than not to be difficult to reduce into a sociocultural meaning.

10 See the related 'sympathetic dissonance' (Bolles and Hunter, 2012, 153-68). 
Collaborating on that process of not-knowing is what I previously called generating a positionality. It is difficult to speak of audience engagements other than my own, but Hernández asked the audience to take the time, when leaving the building to write in a notebook about their feelings. Some found the piece frustrating and did not know what to make of it, or how to articulate its "meaning." Others found it deeply affecting - it changed them. The piece drew them into its fabric, asked them to set aside their individuality, and offered them materials for their own forgetting and remembering, their own selving that recognizes the dead in the change that happens while colaboring as an audience member with the performance. In doing so, they joined into the positionality of the affective politics. Performance that works on sustaining affect into a materiality with which the audience can co-labor is embodying an affective politics that is core to performativity. It is also core to the sociosituated collaboration that generates the positionalities needed to support and sustain longterm social change - through the way difference happens to the self, and the self begins to sense other ways of becoming, knowing, and valuing.

\section{Referencias}

Barad, K. (2001). Re(con)figuring space, time and matter, in M. Dekoven, ed., Feminist Locations: Global and Local, Theory and Practice. Rutgers University Press, New Brunswick, New Jersey and London, 75-109.

Bolles, D. and Hunter, L. (2012). Scoring the Site Particular. International Journal of Daoist Studies, 5, $153-168$.

Hunter, L. (2010). Allegory Happens: Allegory and the Arts post-1960, in R. Copeland and P. T. Struck, eds. Cambridge Guide to Allegory. Cambridge: Cambridge University Press, 266-80.

Hunter, L. (2016). Ways of Knowing and Ways of being known, in L. Hunter, Krimmer, E., Lichtenfels, P. eds. Sentient Performativities of Embodiment: Thinking alongside the Human. Lexington University Presses, 1-18.

Hunter, L. (2018). The Politics of Practice: A rhetoric of performativity. Palgrave, 2019.

Massumi, B. (2011). Semblance and Event: Activist Philosophy and the Occurrent Arts. London: MIT Press, 2011.

Noé, I. (2009). Site Particular, in Mapping Landscapes for Performance as Research, S. Riley and L. Hunter, eds. London: Palgrave, 207-9. 